OPEN ACCESS

Edited by:

Rupesh Raina,

Akron Children's Hospital,

United States

Reviewed by:

Giuseppe Remuzzi,

Mario Negri Pharmacological Research Institute (IRCCS), Italy

R. Morrison Hurley,

University of British Columbia, Canada

*Correspondence:

Dorottya Kelen

dorottya.Kelen@erasme.ulb.ac.be

Specialty section:

This article was submitted to

Pediatric Nephrology,

a section of the journal

Frontiers in Pediatrics

Received: 15 July 2020 Accepted: 07 September 2020

Published: 18 February 2021

Citation:

Kelen D, Chiodini B, Godart V Adams B, Stordeur $P$ and Ismaili $K$

(2021) Case Report: Neonatal

Unexplained HUS Treated With

Complement Inhibitor Eculizumab.

Front. Pediatr. 8:579607.

doi: 10.3389/fped.2020.579607

\section{Case Report: Neonatal Unexplained HUS Treated With Complement Inhibitor Eculizumab}

\author{
Dorottya Kelen ${ }^{1 *}$, Benedetta Chiodini ${ }^{2}$, Valérie Godart ${ }^{1}$, Brigitte Adams ${ }^{2}$, \\ Patrick Stordeur ${ }^{3}$ and Khalid Ismaili ${ }^{2}$
}

${ }^{1}$ Neonatal Department, Hôpital Erasme, Université Libre de Bruxelles, Brussels, Belgium, ${ }^{2}$ Department of Pediatric Nephrology, Hôpital Universitaire des Enfants Reine Fabiola, Université Libre de Bruxelles, Brussels, Belgium, ${ }^{3}$ Belgian National Reference Centre for the Complement System, Laboratory of Immunology, LHUB-ULB, Université Libre de Bruxelles, Brussels, Belgium

Background: Hemolytic uremic syndrome (HUS) is rare in neonates. It is probably an under-recognized condition in the early postnatal period as it presents similarly to the most common perinatal asphyxia and to differentiate the two conditions is challenging. We describe the clinical presentation of a potential new subtype of neonatal HUS triggered by hypoxic-ischemic event. Our patient was successfully treated by a single dose of Eculizumab as early as at 9 days of life.

Case Report: A 35-weeks infant was born with low hemoglobin and subsequently developed respiratory distress, hypotension, and acidosis. Blood transfusion was administered, acidosis corrected, neurological examination remained reassuring. Few hours later he developed renal failure, macroscopic hematuria, hemobilia, thrombocytopenia and coagulopathy refractory to platelet and fresh frozen plasma transfusions. No infection was found. Haptoglobin was non-measurable, and schistocytes present, complement factors C3, C4 and B were low, FBb increased. HUS was suspected. A single dose of Eculizumab ${ }^{\mathrm{TM}}$ was administered on day 9 of life. No genetic mutation of atypical HUS was found. He was discharged with improving renal function and developing cholestasis.

Conclusion: In neonates with hemolytic anemia, thrombocytopenia, hematuria and renal failure, HUS should be suspected. In neonatal HUS Eculizumab should be considered as first-line therapy and discontinuation can be considered if no genetic mutation is found and clinical condition improves. In very young patients, cholestasis could appear as potential side effect of Eculizumab ${ }^{\mathrm{TM}}$.

Keywords: hemolytic uremic syndrome, perinatal asphyxia, eculizumab, neonatal, case report

\section{INTRODUCTION}

Hemolytic uremic syndrome (HUS) is rare in neonates. It has been observed in von Willebrand factor-cleaving protease (ADAMTS13) deficiency, inborn errors of cobalamin absorption and metabolism, and alternative pathway complement system deficiencies (1). Exceptionally it has been related to E. coli shiga toxins (STEC) (2). 
However, neonatal HUS could also be triggered by a hypoxicischemic event and this is probably an under-recognized condition as in the early postnatal period HUS presents similarly to the more common perinatal asphyxia (PA). To differentiate the two conditions is challenging. We describe the clinical presentation of a potential new subtype of neonatal HUS.

Our patient was successfully treated by a single dose of Eculizumab as early as at 9 days of life.

\section{CASE DISCUSSION}

A 35-week 4 days gestational age male infant was born by cesarean section for placenta praevia, with birth weight of 3,080 g. Maternal Kleinhauer and coombs test were negative, and maternal renal function was normal (creatinine $0.4 \mathrm{mg} / \mathrm{dL}$ ). The newborn had good Apgar scores (9/9/9), and normal cord $\mathrm{pH}$, but low $\mathrm{Hb}(11.6 \mathrm{~g} / \mathrm{dL})$. He was pale and developed hypotension with a blood pressure (BP) of 29/16 mmHg. Noninvasive respiratory support was started as he also developed respiratory distress (peripheral oxygen saturation $86 \%$, heart rate $150-170 / \mathrm{min})$. At $1 \mathrm{~h}$ of life, his blood gas showed anemia and mixed acidosis $\left(\mathrm{pH} 6.9, \mathrm{pCO}_{2} 62 \mathrm{mmHg}, \mathrm{BE}\right.$ $-19 \mathrm{mmol} / \mathrm{L}$, lactate $12 \mathrm{mmol} / \mathrm{L}, \mathrm{Hb} 7.8 \mathrm{~g} / \mathrm{dL}$ ). However, neurological criteria for PA justifying therapeutic hypothermia were not met (3). Following administration of cross-matched red blood cell transfusion, his neurological examination was still reassuring, and his breathing, blood pressure and gas analysis normalized (peripheral oxygen saturation $>95 \%$ in air, BP 53/29 mmHg, lactate $1.9 \mathrm{mmol} / \mathrm{l}$ ). At $30 \mathrm{~h}$ of life he developed macroscopic hematuria, oliguria, hypertension, severe renal failure and hyperkalemia $\left(\mathrm{K}^{+} 9.5 \mathrm{mmol} / \mathrm{L}\right.$, urea $71.6 \mathrm{mg} / \mathrm{dL}$, creatinine $3 \mathrm{mg} / \mathrm{dL}$ ). Liver enzymes were elevated (AST $2013 \mathrm{U} / \mathrm{L}$, ALT $625 \mathrm{UI} / \mathrm{L})$, serum LDH increased (9,920 $\mathrm{U} / \mathrm{L})$, while CK levels were normal (820 U/L). He also showed signs of anemia, thrombocytopenia and coagulopathy (Hb 11.5 $\mathrm{g} / \mathrm{dL}$, Plt 23.000/ $\mathrm{mm}^{3}$, INR 2.76, fibrinogen $82 \mathrm{mg} / \mathrm{dL}$, D-dimer $>35,000 \mathrm{ng} / \mathrm{ml} \mathrm{FEU})$. Antibiotics were started in view of the clinical deterioration, although no infection was found. Renal ultrasound showed bilateral cortical hyperechogenicity with no signs of renal thrombosis.

On day 3, cranial ultrasound, amplitude-integrated electroencephalogram, and neurological clinical examination were still reassuring. The infant received a second blood transfusion, fresh frozen plasma (FFP) and platelet transfusion. Then a second platelet transfusion and FFP for 6 consecutive days at a dose of $15 \mathrm{ml} / \mathrm{kg}$ were administered, with no improvement in coagulation or platelet count, and further deterioration of the renal function. His $\mathrm{BP}$ was still elevated with a maximum measured of $90 / 58 \mathrm{mmHg}$. In addition to hematuria, hemobilia was observed without hyperbilirubinemia.

As there was no clear etiology of the multi-organ failure (MOF) and disseminated intravascular coagulopathy (DIC), and

Abbreviations: HUS, Hemolytic uremic syndrome; FFP, Fresh frozen plasma; DIC, Disseminated intravascular coagulopathy; MOF, multiorgan failure; PA, Perinatal asphyxia; AKI, acute kidney injury. neurological criteria for asphyxia were not met, investigations toward HUS have been initiated.

Haptoglobin was non-measurable, his peripheral blood smear test showed signs of hemolysis and schistocytes count was at about $10 \%$. Cobalamin and ADAMTS 13 were within the normal range for age, and ANCA, ANA and anti-FH antibodies were not detected. Concentration of the following components of the complement system (measured $48 \mathrm{~h}$ after FFP administration) appeared decreased: C3 $27 \mathrm{mg} / \mathrm{dL}$ (normal range: 80-164 $\mathrm{mg} / \mathrm{dL}$ ), C4 $5 \mathrm{mg} / \mathrm{dL}$ (normal range: $10-46 \mathrm{mg} / \mathrm{dL}$ ), Factor B $6 \mathrm{mg} / \mathrm{dL}$ (normal range: 11-22 mg/dL), Factor I $3.9 \mathrm{mg} / \mathrm{dL}$ (normal range: 4.0-10.7 mg/dL), and Factor $\mathrm{H} 34 \mathrm{mg} / \mathrm{dL}$ (normal range: $37-73 \mathrm{mg} / \mathrm{dL}$ ). CD46 (MCP) expression was normal. Even though these tests were carried out after FFP administration and the normal values for adults are not adapted to neonates, the observed results suggested an alteration of the alternative pathway, confirmed by a significant increase of the $\mathrm{FBb}$ concentration [FBb $0.68 \mathrm{mg} / \mathrm{dL}(<0.15)]$. Genetic test results for atypical HUS showed eventually no mutations in $\mathrm{CFH}, \mathrm{CFI}, \mathrm{CFB}, \mathrm{C} 3, \mathrm{CD} 46$ (MCP), Thrombomodulin, CFHR1-R3 deletion, Recombinant CFH/CFHR1 hybrid, and DGKE genes.

In this clinical situation, on day 9 of life, $300 \mathrm{mg}$ of Eculizumab $^{\mathrm{TM}}$ were administered. Antibiotic prophylaxis was given, and vaccination completed as soon as 8 weeks of age. Kidney function started to improve 3 days after the administration of Eculizumab, while coagulation and platelet count improved between days 5 and 14 (Figure 1). Gammaglutamyl transferase (GGT) levels, which were initially normal, started to increase.

The baby was discharged on day 29 of life with improving renal function (urea $78.4 \mathrm{mg} / \mathrm{dL}$, creatinine $1.64 \mathrm{mg} / \mathrm{dL}$ ), and developing cholestasis. At 3 months of life, he underwent endoscopy resolving his cholestasis, his BP at that time was $115 / 55 \mathrm{mmHg}$.

At 10 months of life, he showed an improved renal function (urea $30 \mathrm{mg} / \mathrm{dL}$, creatinine $0.53 \mathrm{mg} / \mathrm{dL}$ ), and normalized values of hematology ( $\mathrm{Hb} 10.2 \mathrm{~g} / \mathrm{dL}$, Plt $\left.449 \times 10^{3} / \mu \mathrm{L}\right)$, liver enzymes (GOT 35 UI/L, GPT 26 UI/L, Serum bilirubin $<0.2 \mathrm{mg} / \mathrm{dL}$, GGT $24 \mathrm{UI} / \mathrm{L}$ ), and complement components (C3 $0.82 \mathrm{~g} / \mathrm{L}, \mathrm{C} 40.15$ g/L, FBb 0.1 mg/dL, Haptoglobin 130 mg/dL LDH 269 UI/L, schistocytes 4/1,000 GR).

\section{DISCUSSION}

In neonates presenting with acute kidney injury (AKI), MOF and coagulopathy, it is often difficult to distinguish between DIC, PA, and neonatal HUS. Few elements can help in the differential diagnosis.

Usually PA manifests with neurological signs directly after birth, while symptoms of MOF and DIC develop subsequently (1). In HUS and sepsis neurological signs develop further to MOF and DIC. Gross hematuria is often an early sign in neonatal HUS, as well as severe thrombocytopenia $(4,5)$. Schistocytes are classically present in HUS and are generally low or not found in the other conditions (2) (Table 1). 


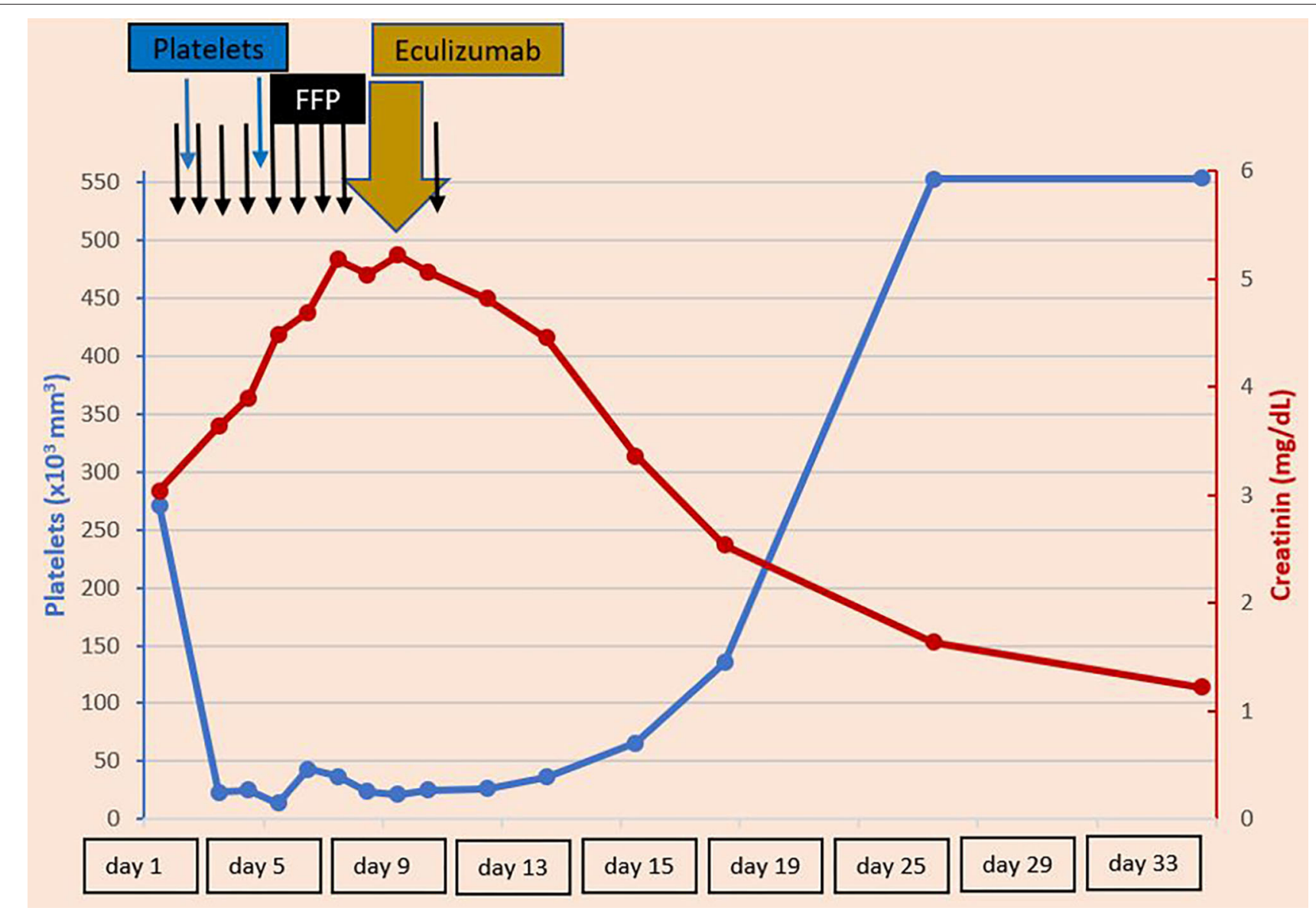

FIGURE 1 | Platelets and creatinine levels during the first 33 days of patient's life-Refractory thrombocytopenia and severe renal failure developed on day 2 of life. Improvements have been observed after administration of Eculizumab on day 9 of life. FFP, Fresh frozen plasma.

TABLE 1 | Elements to help in the differential diagnosis between perinatal asphyxia (PA), disseminated intravascular coagulation (DIC), and neonatal hemolytic uremic syndrome (HUS).

\begin{tabular}{lccc}
\hline Characteristics & PA & DIC & Neonatal HUS \\
\hline Haemolytic anemia & - & $-/+$ & +++ \\
Increased level of LDH/CK & $++/+++$ & $+/-$ & $+++/-$ \\
Thrombocytopenia & - & ++ & +++ \\
Schistocytes & - & $+/-$ & +++ \\
AKI & $+/-$ & - & +++ \\
Haematuria & - & $+/-$ & +++ \\
Coagulopathy & $+/-$ & +++ & +++ \\
Early neurological signs & +++ & $-/+$ & $-/+$ \\
Alteration of complement system & - & $+/-$ & +++ \\
Blood pressure & $\downarrow / \uparrow$ & $\downarrow$ & $\uparrow \uparrow$ \\
\hline
\end{tabular}

Our patient presented with anemia, thrombocytopenia and AKI without neurological signs, and soon developed gross hematuria with extremely high D-dimers. Altogether it was not a typical picture of PA.
A hypoxic-ischemic perinatal event, in our case hypotension due to possible acute blood loss from the placenta previa, might have caused endothelial cell damage leading to a vicious circle of consumption of platelets and plasma factors which then triggered HUS. The endothelial cell injury caused by ischemia could have been the starting point of the microangiopathic cascade. Biran et al. have hypothesized a similar mechanism on three neonates who developed renal failure with biological features compatible with HUS following a hypoxic-ischemic perinatal event (1). Our diagnosis was based on extremely high $\mathrm{FBb}$ levels demonstrating complement activation (6), although interpretation of complement results was compromised by previous FFP administration and reference intervals adopted to adults but not to neonates. Concentration of most of the complement factors is indeed lower in neonates compared to children and adults, and even lower in preterm neonates (7). This is especially the case of FH, FI and FB (8).

Absence of well-defined reference intervals for neonates is not the only problem to face in complement testing. The other main drawback is the absence of standardization of the techniques used to measure the parameters of the complement system (7). 
Since 2011, Eculizumab, a recombinant human monoclonal anti-C5 antibody is approved by FDA for the treatment of atypical HUS (9).

An international group of experts suggests Eculizumab as first-line treatment for children with clinical diagnosis of atypical HUS even when no genetic mutations are found (10).

Eculizumab has been successfully used in a few cases of neonatal HUS, most of them presenting a factor $\mathrm{H}$ mutation $(4,5$, 11, 12). In all cases Eculizumab has been administered long term, usually on a regime of $300 \mathrm{mg}$ every 3 week. Our patient received $300 \mathrm{mg}$ Eculizumab on day 9 of life. To our best knowledge, he is the youngest newborn with HUS successfully treated with the anti-C5 monoclonal antibody. In view of his improving clinical condition, and the absence of a consensus about the duration of the therapy without underlying genetic condition, we stopped the treatment after a unique dose of Eculizumab (10). The risks of prolonged Eculizumab therapy are not negligible and include immunosuppression, need for prophylactic antibiotics, and high financial costs.

The appearance of cholestasis, observed few days after Eculizumab administration is a potential side effect already described in young STEC-HUS cases (13). It is still uncertain if the delayed cholestasis is a side effect of Eculizumab itself or an unusual complication of the HUS disease. However, Mauras et al. suggest that this risk should be kept in mind in patients receiving Eculizumab at a very young age (13).

\section{CONCLUSION}

In neonatal cases with history of a hypoxic- ischemic event, presenting with hemolytic anemia, thrombocytopenia, gross hematuria and AKI, HUS should be suspected. In this scenario, genetic tests toward atypical HUS are required, and complement factors should be analyzed before FFP administration. In case of neonatal HUS Eculizumab should be considered as firstline therapy and FFP administered until the anti-C5 antibody is available. Liver enzymes should be monitored following the administration of Eculizumab. In HUS triggered by perinatal hypoxic- ischemic events when no genetic mutations are found,

\section{REFERENCES}

1. Biran V, Fau S, Jamal T, Veinberg F, Renolleau S, Gold F, et al. Perinatal asphyxia may present with features of neonatal atypical hemolytic uremic syndrome. Pediatr Nephrol. (2007) 12:2129-32. doi: 10.1007/s00467-007-0570-7

2. Ulinski T, Lervat C, Ranchin B, Gillet Y, Floret D, Cochat P. Neonatal hemolytic uremic syndrome after mother-to-child transmission of Escherichia coli O157. Pediatr Nephrol. (2005) 20:1334-5. doi: 10.1007/s00467-005-1871-3

3. Azzopardi D, Brocklehurst P, Edwards D, Halliday H, Levene M, The TOBY Study Group, et al. Whole body hypothermia for the treatment of perinatal asphyxial encephalopathy. A randomised controlled trial. BMC Pediatr. (2008) 8:17. doi: 10.1186/1471-2431-8-17

4. Anastaze Stelle K, Gonzalez E, Wilhelm-Bals, Michelet PR, Korff CM, Parvex P. Successful treatment of neonatal atypical haemolytic uremic syndrome with C5 monoclonal antibody. Arch Pédiatr. (2016) 23:2836. doi: 10.1016/j.arcped.2015.11.020

5. Besbas N, Gulhan B, Karpman D, Topaloglu R, Duzova A, Korkmaz E, et al. Neonatal onset atypical hemolytic uremic syndrome stopping Eculizumab therapy can be considered in case of improving clinical conditions.

\section{DATA AVAILABILITY STATEMENT}

The original contributions presented in the study are included in the article/supplementary material, further inquiries can be directed to the corresponding author/s.

\section{ETHICS STATEMENT}

Ethical review and approval was not required for the study on human participants in accordance with the local legislation and institutional requirements. Written informed consent to participate in this study was provided by the participants' legal guardian/next of kin. Written informed consent was obtained from the minor(s)' legal guardian/next of kin for the publication of any potentially identifiable images or data included in this article.

\section{AUTHOR CONTRIBUTIONS}

DK: physician in charge of the clinical care of the case, drafting the manuscript, revising the manuscript, final approval of the version to be published. BC: drafting the manuscript, revising the manuscript, final approval of the version to be published. VG: drafting the case report in the manuscript, final approval of the version to be published. BA: revising the manuscript, final approval of the version to be published. PS: complement factors measurements, analysis and interpretation of data, revising the manuscript, final approval of the version to be published. KI: specialist contribution to the clinical treatment, revising the manuscript, final approval of the version to be published. All authors contributed to the article and approved the submitted version.

\section{ACKNOWLEDGMENTS}

The authors are grateful for the intellectual support of Miklós Szabó and Nora Tusor.

successfully treated with eculizumab. Pediatr Nephrol.

(2013) 28:155-8. doi: 10.1007/s00467-012-2296-4

6. Cataland SR, Holers VM, Geyer S, Yang S, Wu HM. Biomarkers of terminal complement activation confirm the diagnosis of aHUS and differentiate aHUS from TTP. Blood. (2014) 123:3733-8. doi: 10.1182/blood-2013-12547067

7. Grumach AS, Ceccon ME, Rutz R, Fertig A, Kirschfink M. Complement profile in neonates of different gestational ages. Scand J Immunol. (2014) 79:276-81. doi: 10.1111/sji.12154

8. De Paula PF, Barbosa JE, Junior PR, Ferriani VP, Latorre MR, Nudelman V, et al. Ontogeny of complement regulatory proteins concentrations of factor $\mathrm{H}$, factor $\mathrm{I}$, c4b-binding protein, properdin and vitronectin in healthy children of different ages and in adults. Scand J Immunol. (2003) 58:572-7. doi: 10.1046/j.1365-3083.2003.0 1326.x

9. Soliris (eculizumab) Full Prescribing Information. Cheshire, CT: Alexion Pharmaceuticals (2011).

10. Loirat C, Fakhouri F, Ariceta G, Besbas N, Bitzan M, Bjerre A, et al. An international consensus approach to the management of atypical 
hemolytic uremic syndrome in children. Pediatr Nephrol. (2016) 31:1539. doi: 10.1007/s00467-015-3076-8

11. Sharma S, Pradhan M, Meyers KE, Le Palma K, Laskin BL. Neonatal atypical hemolytic uremic syndrome from a factor $\mathrm{H}$ mutation treated with eculizumab. Philadelphia Clin Nephrol. (2015) 84:181-5. doi: $10.5414 / \mathrm{CN} 108532$

12. Michaux K, Bacchetta J, Javouhey E, Cochat $P$, Frémaux-Bacchi V, Sellier-Leclerc AL. Eculizumab in neonatal hemolytic uremic syndrome with homozygous factor H deficiency. Pediatr Nephrol. (2014) 29:24159. doi: 10.1007/s00467-014-2933-1

13. Mauras M, Bacchetta J, Duncan A, Lavocat MP, Rohmer B. Escherichia coliassociated haemolytic uremic syndrome and severe chronic hepatocellular cholestasis: complication or side effect of eculizumab? Pediatr Nephrol. (2019) 34:1289-93. doi: 10.1007/s00467-019-04234-6
Conflict of Interest: PS has received fees from Alexion Pharmaceuticals through his employer.

The remaining authors declare that the research was conducted in the absence of any commercial or financial relationships that could be construed as a potential conflict of interest.

Copyright $\odot 2021$ Kelen, Chiodini, Godart, Adams, Stordeur and Ismaili. This is an open-access article distributed under the terms of the Creative Commons Attribution License (CC BY). The use, distribution or reproduction in other forums is permitted, provided the original author(s) and the copyright owner(s) are credited and that the original publication in this journal is cited, in accordance with accepted academic practice. No use, distribution or reproduction is permitted which does not comply with these terms. 